\title{
EVIDENCE COLLECTED FOR PEER REVIEW OF BUCKLING-RESTRAINED BRACED FRAMES IN NEW ZEALAND
}

\author{
Dan Court-Patience ${ }^{1}$ and Mark Garnich ${ }^{2}$
}

(Submitted June 2020; Reviewed September 2020; Accepted March 2021)

\begin{abstract}
Buckling-restrained braces (BRBs) form a bracing system that provides lateral strength and stiffness to a building. These systems have been shown to provide larger energy dissipation in severe earthquake events compared to concentrically and eccentrically braced frames (CBFs and EBFs). However, unlike CBFs and EBFs there is no guidance document or specific instructions in regulatory standards for the design of buckling-restrained braced frames (BRBFs) in New Zealand. This makes it difficult for structural engineers to be aware of all the strength and stability considerations required for the safe design of BRBFs. Currently, structural designs that include BRBFs require a peer-review to gain building compliance. The American standard ANSI/AISC 341-16 is the adopted document used in New Zealand for guidance in how to collect evidence showing a BRBF system will perform as intended. However, as ANSI/AISC 341-16 is not a governing document in New Zealand, instructions within the document are not enforced and can be made to fit within the constraints of a building project. By way of example, this paper presents the experimental test process and results acquired from pre-qualification testing of three different commercially available BRB architypes. Of the three BRB designs investigated, one failed prematurely due to global buckling. A manufacturing error was the likely cause of this premature failure. This failure highlights the need for strict quality control during fabrication. All remaining BRBs performed well, meeting the acceptance criteria set out in ANSI/AISC 341-16. Positive pre-qualification results meant the BRBs were installed in medium to high-rise buildings throughout New Zealand. The importance of sub-assemblage testing to assess the performance of a BRB and its frame components is also discussed. Finally, the capability of high fidelity modelling to supplemental physical testing is also presented.
\end{abstract}

\section{BACKGROUND}

Buckling-restrained braces (BRBs) are a critical component in providing a building with lateral stability and absorbing energy in strong seismic events. They are unique as they perform almost evenly in compression and in tension. This means larger energy dissipation than global buckling and bending behaviour exhibited in concentrically and eccentrically braced frames (CBFs and EBFs). First developed in Japan in the late 1970s, they gained rapid acceptance in the US after the 1994 Northridge Earthquake [1-4]. In general, all BRBs work on the same mechanical principles. Primarily a slender steel core plastically deforms inside a restrainer. In tension, the core stretches, exhibiting strain hardening and a reduction in cross sectional area. In compression, the specimen is also configured for axial ideal loading. However, the slender nature of the steel tendon and de-bonded contact with the restrainer causes constrained buckling. This generally progresses through several modes of buckling as compressive uniaxial displacement of the specimen increases. These two behaviours (axial compressive and bending due to constrained buckling) are superimposed. The inherent design of the restrainer encasing the yielding section of the core makes it difficult to observe the deformation of the core. The highly non-linear behaviour of the BRB core makes it challenging to predict the force-displacement response and rotational demands along the length of a BRB. Predicting the force-displacement response and rotational demands is important to ensure ductile design of the system while restricting unwanted behaviour such as weld tearing and connection zone failure.
As BRBs are relatively new to the New Zealand market there is not yet any documented guidance or specific instructions in regulatory standards for the design of BRBFs. This makes it difficult for engineers to anticipate all the possible stability and strength issues within a system and actively mitigate them in each design. To help ensure BRBF designs perform as intended, a peer review and physical testing are currently required to gain building compliance in New Zealand. The American standard of seismic provisions for structural steel buildings ANSI/AISC 341-16 [5] is the commonly adopted document that describes how to conduct testing and present evidence to show that each BRB system will perform as required. Firstly, BRB prequalification testing is conducted to ensure each BRB specimen is suitably designed and manufactured to perform effectively in design level earthquake events. This type of testing isolates the performance of a BRB by applying a cyclic uniaxial loading protocol with displacement increments based a building projects design story drift.

Results from pre-qualification should show the brace can reach displacements equal to two times the design story drift and cumulative inelastic axial ductility of 200 times displacement at yield strain without failure. Testing also determines the yield strength and over-strength factors required for the design of the connecting elements (beam, column, gusset plate). Other protocols used for brace pre-qualification testing worldwide include EN 15129 as a part of the European Standards [6], which use similar displacement targets as ANSI/AISC 341-16. Whereas, recommendations made by European Convention for Constructional Steelwork [7] specify displacement targets 
based on increments of yield strain instead of design story drift. Other countries such as in Japan are again different, where target displacements are categorised as average strain, this being a percentage of the yielding length of the core. The advantage of using average strain as a basis for target displacements is that a BRB can be tested and approved for use within a set strain-range limit. Then for each specific building project, the structural engineer can simply check if the demand falls within the approved strain range by dividing the design story drift target by the yielding length. The overstrength factors required to design BRB connecting elements can be determined by interpolating between data points from the original testing. This approach puts more onus on the suppliers up front, but means testing for each building project is not required. Rather, each customer takes advantage of the initial testing.

The financial cost and limited availability of capable laboratories mean testing of every BRB architype and variation is not viable. Commonly, BRB manufactures test a selection of BRBs and extrapolate results between sizes. However, the complex behaviour exhibited in BRBs makes it difficult to interpolate results between different BRB sizes accurately.

Due to the confidential and proprietary nature of BRBs, test data from pre-qualification testing are rarely made available to the public. Published test data of BRBs include five BRBs designed by Nippon Steel. These had post-yield capacities between $1800 \mathrm{kN}$ and $2700 \mathrm{kN}$ and core lengths of $4500 \mathrm{~mm}$. This testing was conducted by the Pacific Earthquake Engineering Research Center in Taiwan. The test report also outlines several tests conducted in Japan and at UC Berkeley [8]. In addition to these groups, Star-Seismic conducted BRB testing on two different BRB specimens. The testing was conducted at Budapest University of Technology and Economics as stipulated by EN 15129 standards. Results showed that the two brace specimens performed well [9]. The average yield strength was $240 \mathrm{kN}$ and testing of the BRB occurred vertically. Another experimental investigation of a bolted and pinned BRBF was undertaken at the University of Auckland [10]. The experimental test results showed stable hysteresis loops. Again, brace design yield capacities were low at $200-250 \mathrm{kN}$.

Global stability is another main consideration when examining BRBF behaviour. Evidence following the 2011 Tōhoku Earthquake [11] and from several full frame tests have shown BRBFs to perform well [12-17]. However, the complexity of BRB behaviour makes it difficult to verify frame stability for all configurations and sizes. ANSI/AISC 341-16 recommends sub-assemblage testing as well as pre-qualification testing to take place. Sub-assemblage testing aims to ensure there will be no instability or premature failure as the BRB interacts with the other frame elements. Japan has developed an alternative approach to sub-assemblage testing by including in their design codes analytical methods to assess stability that considers the BRB, connection zone and frame elements. These methods assume conditions stipulated by pre-qualification specifications that BRB designers have to meet [18]. In Taiwan, designs follow a similar method to safeguard against instability [19], but only provide instructions for BRBs with welded connections. In both Japan and Taiwan, the amount of variation in the design of connections is limited to proven cases and clear instructions are given to structural engineers on how to incorporate BRBs. This generally means the connection zone is required to be relatively rigid and often stiffened. Internationally, few design codes have this governance, which has led to designs that fall outside of already proven cases and make it difficult to prove stability without physical testing. This is particularly important, as it is unrealistic to expect structural engineers to understand all the possible stability issues and apply them to their designs. Sub-assemblage testing can consist of partial systems, full-frame or complete story specimens. This is recommended as each seismic frame design is bespoke and the available test data for these systems is scarce. Market rates for accredited uniaxial testing are approximately $\$ 8-10 \mathrm{k}$ (USD) depending on testing demand, with sub-assemblage testing costing more. In New Zealand, the financial pressures inherent in commercial projects has led to uniaxial testing being favoured without sub-assemblage testing.

Alternatively, BRB suppliers instil confidence by showing results from other sub-assemblage tests that demonstrate performance and stability. However, the low regulation in New Zealand allows for a large variation of connections zones in BRBFs, with connections often being more flexible than of those tested. Also, unlike Japan and Taiwan who often use BRBFs as a supplemental damper, New Zealand uses BRBFs as the primary lateral support system. There is a level of uncertainty regarding the seismic stability of these systems. Without the limitations imposed on BRBF design, such as those in Japan and Taiwan, project specific sub-assemblage testing and pre-qualification should take place. In addition, the confidential and proprietorial nature of commercial building and BRB designs limit the availability of shared BRB test data making it difficult for the New Zealand engineering community to determine best practice.

\section{INTRODUCTION}

By way of example, this paper presents the experimental test process and results of three BRBs. As part of a peer review, these tests provided the evidence needed to gain building compliance. This information is valuable since test data for BRBs relevant to the New Zealand construction environment are not publically available, with many research projects restricted to the study of relatively small braces.

\section{Composition and Manufacturing of a BRB}

A BRB is a fabricated assembly. BRBs commonly consist of a ductile-grade steel core plate designed as the main yielding section. A steel tube encases the core, which acts as permanent formwork for grout contained within. To restrict excessive movement of the restrainer in relation to the centre of the brace, a shear key resides in the centre of the core. The core is intentionally de-bonded from the grout and casing to promote independent movement. This is achieved by wrapping the core in a thin film-like material that creates a low friction gap between the core and the restrainer. The core area is sized for the design level seismic load using the cross-sectional area of the core multiplied by the material yield strength. Other types of cross-sections, such as cruciform or multiple plates are also used. Shown in Figure 1 is a schematic of the key components of a BRB that has pinned end connections. Not shown is the concrete that fills the space between the core and the casing. The polystyrene spacers allow for core movement independent of the restrainer. Figure 2 presents the assembly of a BRB from different views.

BRBs appear simple enough, however BRBs display non-linear behaviour inside the restrainer that is difficult to predict, particularly in compression. Performance and stability conditions are sensitive to changes in geometry, material and construction. In the manufacturing of a BRB, the end connections and core are cut from one piece of steel. These parts are welded together. A debonding material is applied to the external surface of this welded section. A steel casing is made by folding or bending a sheet of steel to form the desired shape. Polystyrene spacers are attached to the tapered zones of the welded core section. The spacers create a zone free of concrete that allows the core to deform under compressive loading without pressing directing onto the restrainer. This reduces damage to the concrete restrainer. The welded core section is placed in the centre of the casing and fixed in place with an end cap. Once in place, cement-based grout is poured in the mould 
made by the core and steel casing. The restrainer is then sealed by the other end cap. Once the grout has cured the BRB is complete.

In general, all BRBs work on the same mechanical principles. However, designs vary due to form, function and cost. For example, the geometric dimensions are varied to fit the frame dimensions of each construction project, and connections types vary depending on the structural design strategy, cost and construction environment.

\section{BRB Specimens Tested}

Presented are the test results of three brace designs. Each BRB investigated was assigned a Brace ID during testing. The BRBs selected serve to encompass a variety of BRB options on the market. The braces presented in this paper represent the only braces commercially manufactured in New Zealand. Table 1 presents the details of the braces investigated and 3D drawings of each brace are presented in Figure 3-5.

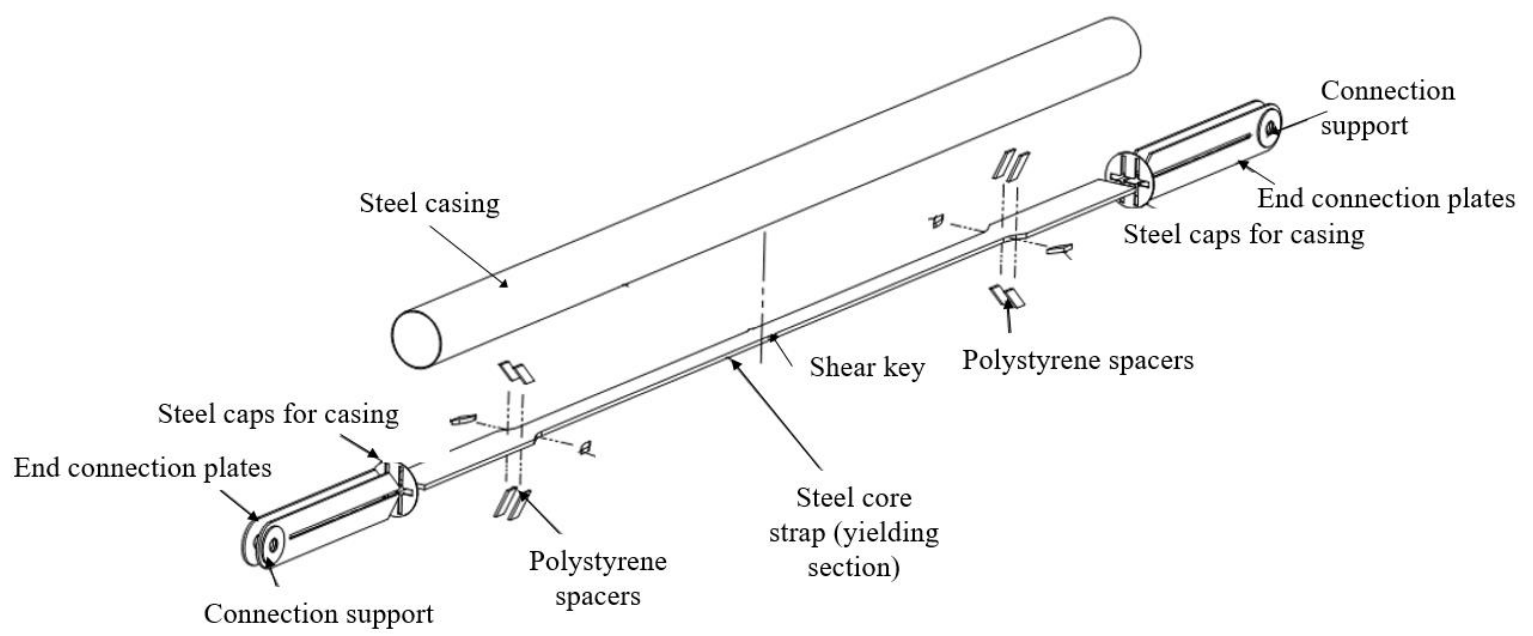

Figure 1: Example of components for a BRB.

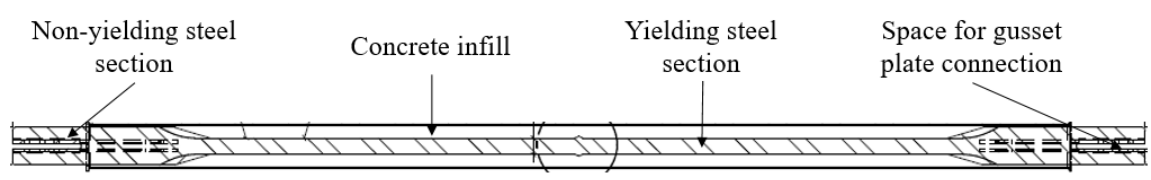

(a) Plan view

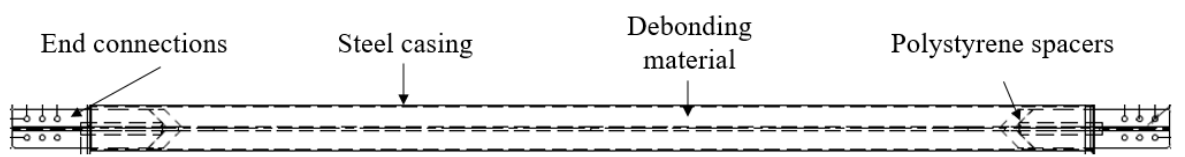

(b) Side elevation

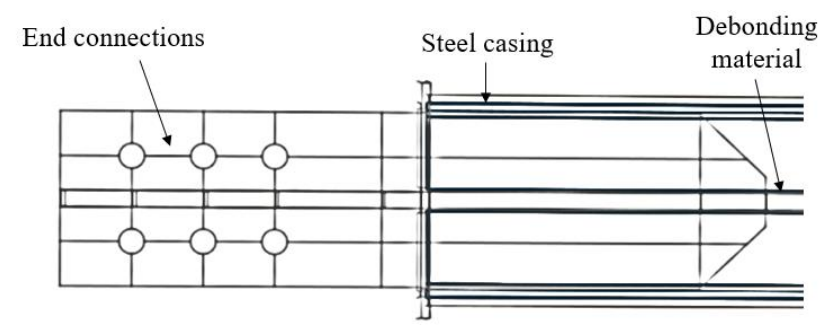

(c) Side elevation (close up with end connection)

Figure 2: Example of components for a BRB.

Table 1: Details of BRB specimens investigated.

\begin{tabular}{cccccc}
\hline Brace ID & $\begin{array}{c}\text { Total Length } \\
(\mathbf{m m})\end{array}$ & Connection type & $\begin{array}{c}\text { Installed angle of } \\
\text { inclination }\left(\boldsymbol{(}^{\mathbf{0}}\right)\end{array}$ & $\begin{array}{c}\text { Core Area } \\
(\mathbf{m m} \mathbf{x} \mathbf{~ m m})\end{array}$ & $\begin{array}{c}\text { Design Yield Force } \\
(\mathbf{k N})\end{array}$ \\
\hline BRB 6.5a & 7238 & Pinned & 24.7 & $25 \times 140$ & 1222 \\
\hline BRB S3 & 3812 & Bolted & 43.15 & $12 \times 55$ & 204 \\
\hline BRB C6 & 6566 & Pinned & 32.9 & $50 \times 85$ & 1445 \\
\hline
\end{tabular}



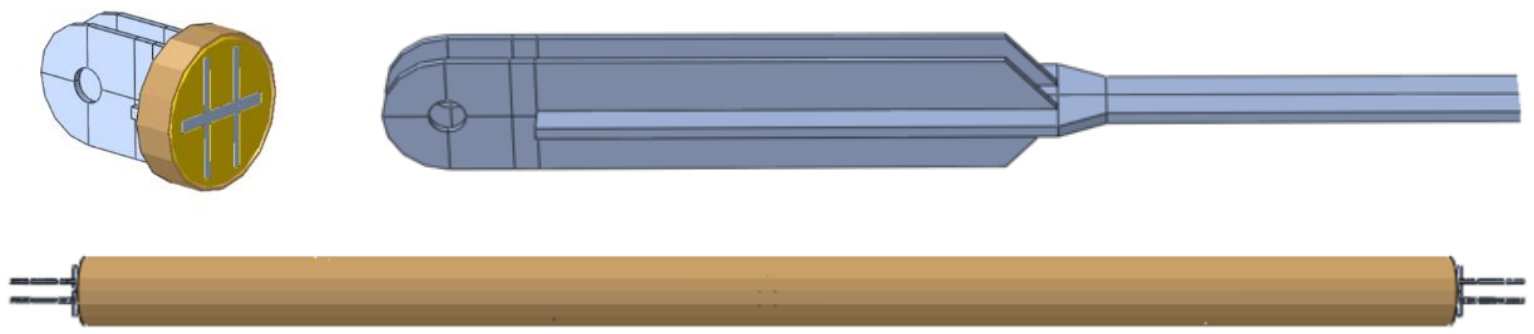

Figure 3: 3D Depiction of BRB 6.5a.
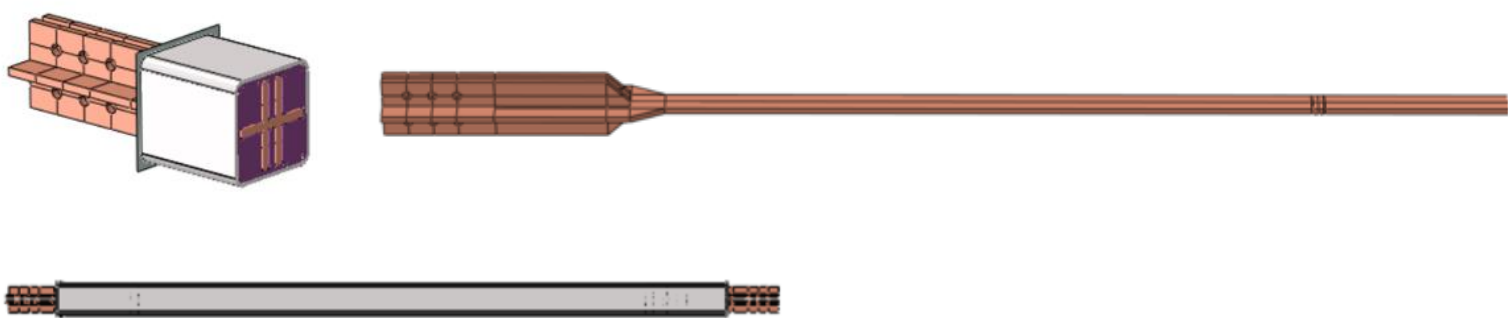

Figure 4: 3D Depiction of BRB S3.
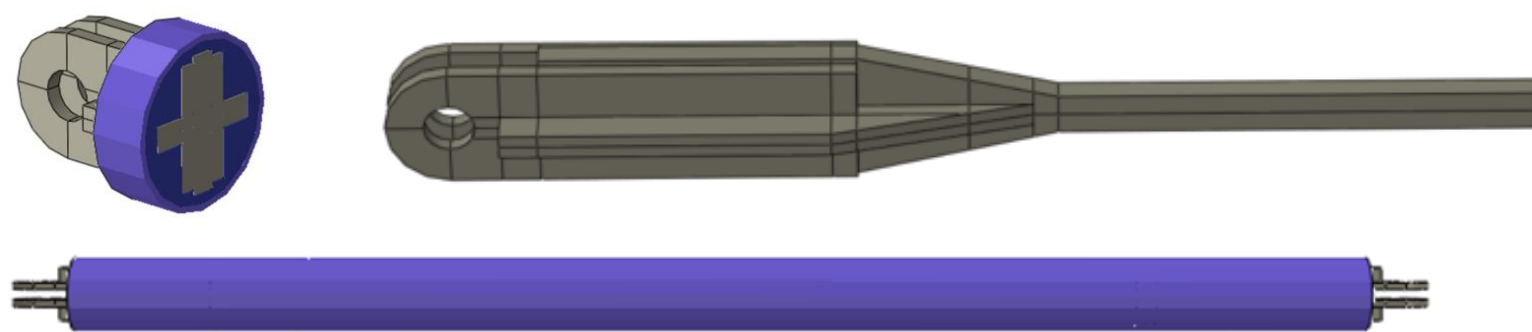

Figure 5: 3D Depiction of BRB C6.

The testing of the specimens described in Table 1 took place within an IANZ accredited testing laboratory. The test apparatus (Figure 6) represented the connection details and load paths of the actual brace installation as close as practicable. An example of a bolted connection used in the test apparatus is shown in Figure 7.

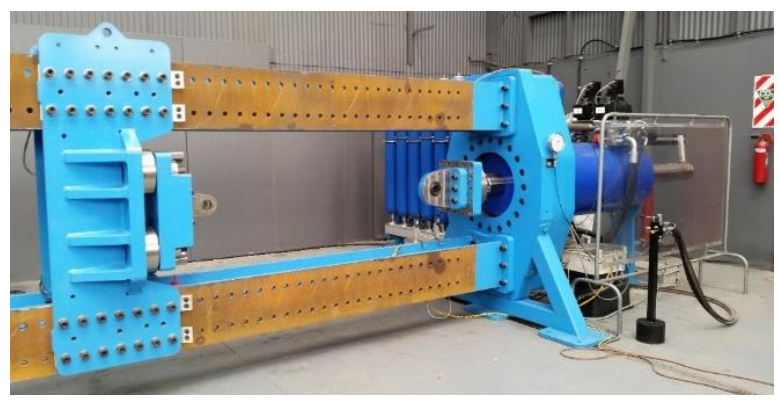

Figure 6: A tension/compression capable horizontal test machine.

Each BRB was oriented in a horizontal direction during testing. This orientation induces an initial curvature due to flexure of the BRB under gravity, similar to in-service conditions. Testing involved imposing displacements to each $\mathrm{BRB}$ in predetermined increments, up to a maximum displacement equivalent to two times the design story drift of the intended structural frame.

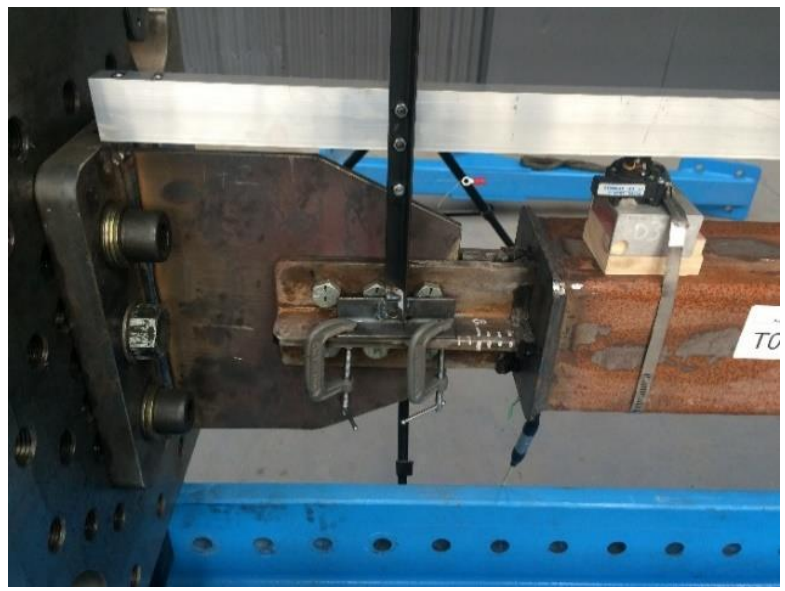

Figure 7: Gusset connection as installed in test apparatus.

\section{EXPERIMENTAL TESTING METHODOLOGY}

Uniaxial testing was used to assess the performance of each BRB specimen. This type of cyclic testing acts to induce a continually increasing axial strain within the BRB core, without the application of a bending moment. Test loading occurs in a cyclic manner, inherently subjecting the BRB core to varying cycles of tensile and compressive strains. 
The gusset plates were bolted to mounting platens within the test apparatus. An actuator is extended to a position where the BRB could be installed without preload. Imposed displacements, based upon the design story drift (where each $\mathrm{BRB}$ was intended for), were applied to one end of the BRB via a hydraulic actuator in accordance with the loading protocol.

\section{Loading Protocol}

Test loading was applied in accordance with ANSI/AISC 34116 Section K3 'Cyclic Tests for Qualification of Buckling Restrained Braces', with loading as prescribed in Section K3.4.c 'Loading Sequence' [5].

During the first load cycle of the test, an initial displacement is made in a positive drift direction (tension) until the force verse displacement response plot exhibited the occurrence of yielding. Yielding is defined as a distinct reduction in the stiffness. Once the initial tension yield displacement was discovered, the direction of loading was reversed in the opposite direction to an equal value of force (induced compression).

All subsequent cycles of loading are applied to pre-determined levels of displacement in both the tension and compression loading directions. At each level of drift displacement, the specimen was cycled from tension into compression for two complete cycles before incrementing to the next drift displacement level. At the request of the engineer of record, additional cycles were applied to BRB S3 and BRB C6. Figure 8 displays an example of loading regime prescribed in [5].
The displacement amplitudes of each loading cycle are dependent on multiples of design story drift that each brace is designed for. The following notations are used to represent the level of deformation and load each brace experiences.

$\Delta_{b} \quad$ The level of displacement, drift imposed on the test specimen.

$\Delta_{b y}$ The level of displacement, $\Delta_{b}$ (drift), at first significant yield of the test specimen.

$\Delta_{b m}$ The level of deformation, $\Delta_{b}$ (drift), corresponding to the design story drift.

\section{Test Procedure}

Cyclic testing was performed in accordance with the loading protocol, beginning by putting the brace into tension. The displacement of the actuator was computer servo controlled. A string pot displacement sensor recorded displacement of the brace. A four-cell load array at one end of the test rig recorded the axial force throughout each test. To capture an accurate yield point and to reduce post yield displacement on the first cycle an initial rate of displacement of $0.5 \mathrm{~mm} / \mathrm{sec}$ was used. Following this first target, the remaining amplitude targets did not require real-time interpretation, so the loading rate was increased to $5 \mathrm{~mm} / \mathrm{sec}$ to reduce overall testing time, while remaining at a pseudo-static rate. An image of BRB C6 installed in the testing apparatus prior to testing is shown in Figure 9.

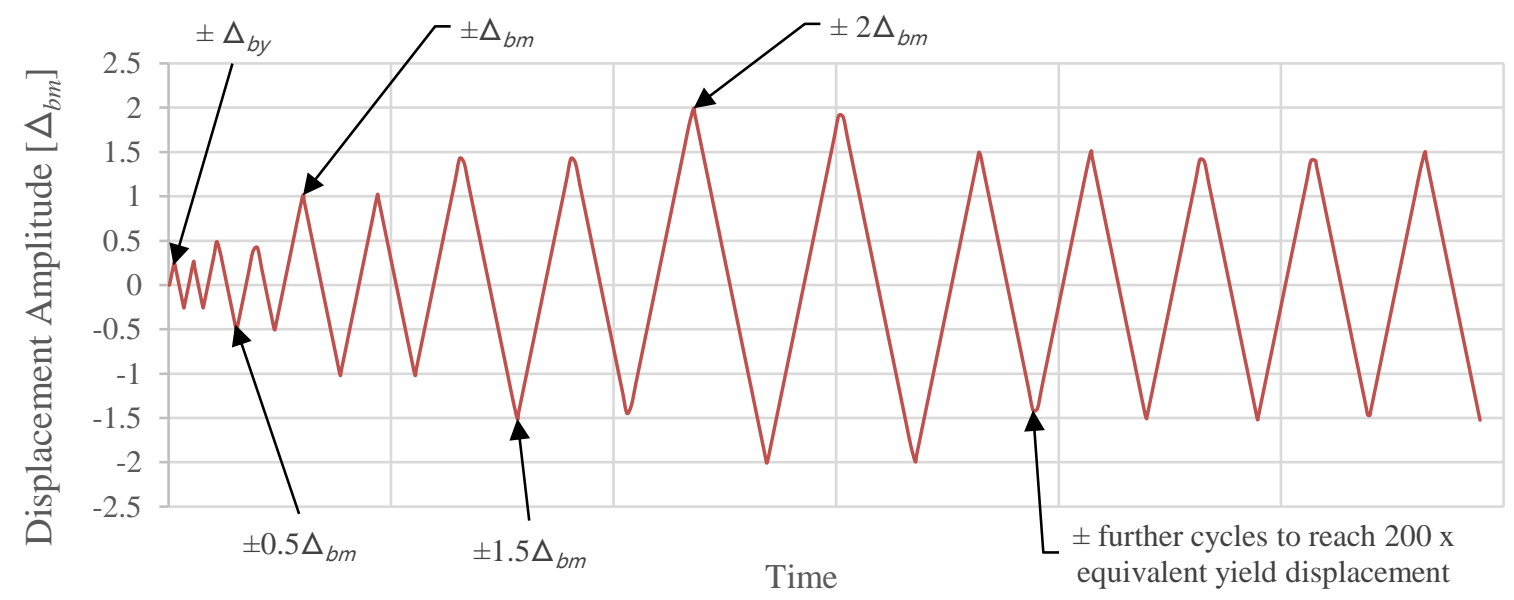

Figure 8: Loading regime for uniaxial cycle BRB testing as per ANSI/AISC 341-16 Manual.

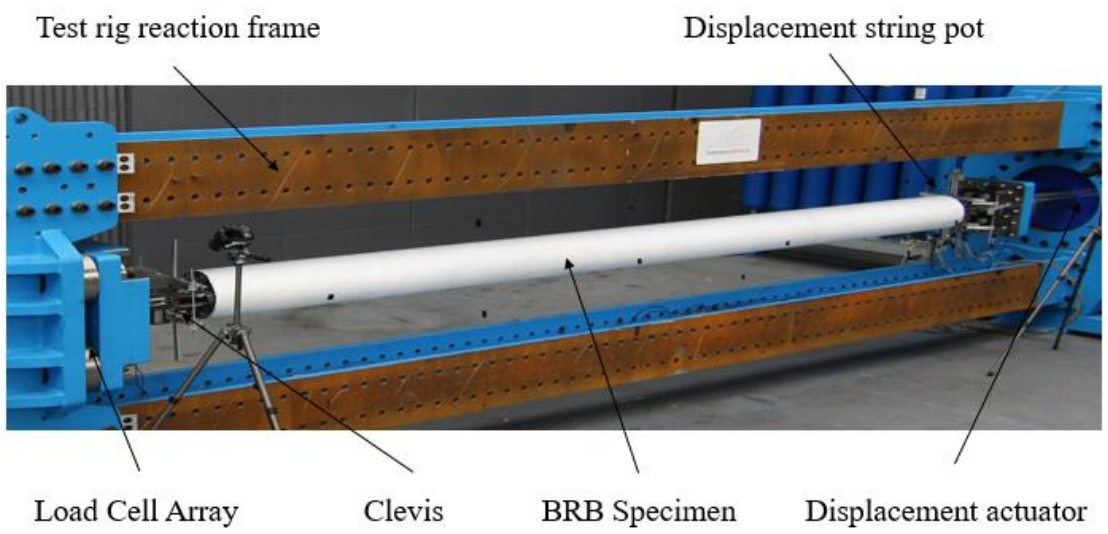

Figure 9: Physical specimen (BRB C6) in testing apparatus. 


\section{EXPERIMENTAL RESULTS}

The force response at peak displacement for each cycle is an important feature of a BRB hysteresis. Results should demonstrate positive tangent stiffness. To ensure yielding of the BRB core and to restrict unwanted behaviour such as weld tearing and connection zone failure, design loads for adjacent frame elements are based on the post-yielding strength of the BRB core. These design loads are determined from prequalification testing. Yield strength and area of the BRB core, are multiplied by adjustment factors accounting for strain hardening $(\omega)$ in tension and strain hardening and compressive effects $(\omega \beta)$ in compression. These factors are based on the BRBs force response to displacements equivalent to two times the design story drift of the frame the BRB is intended for. Figure 10 displays an example force-displacement plot and highlights how the adjustment factors are calculated.

\section{Testing Criteria and Acceptance}

The complete testing methodology and results of testing are enclosed in commercial test reports. This paper provides data otherwise not available. The testing followed requirements set out in ANSI/AISC 341-16. Presented here is the purpose and acceptance criteria used for pre-qualification testing of the BRBs described in Table 1.

Pre-qualification testing serves several purposes. Firstly, it confirms the BRBs yield load and adjustment factors required for design. Secondly, it aims to show how the BRB will perform in a design level earthquake event. Acceptable performance is demonstrated when stable hysteretic behaviour is displayed without failure during the loading protocol in Figure 8. Specifically, results are judged according to the following acceptance criteria as set out in ANSI/ASIC 341-16:

- Display stable, repeatable load - displacement history with positive incremental stiffness.

- No rupture, brace instability or brace end connection failure during testing protocol.

- The maximum tension and compression force during each subsequent cycle after yield shall be no less than the yield force.

- The ratio of maximum compression to tension force during each subsequent cycle after yield shall not exceed 1.3.

\section{Test Results}

The loading protocol and results for the three BRBs (Table 1) are presented in Figure 11-18. The loading protocol for each test is shown in terms of displacement, and displacement relative to design story drift $\left(\Delta_{b m}\right)$. This is followed by the force response of each specimen to its displacement loading protocol. For comparison the force response is also shown in terms of the overstrength ratio (force/yield force).

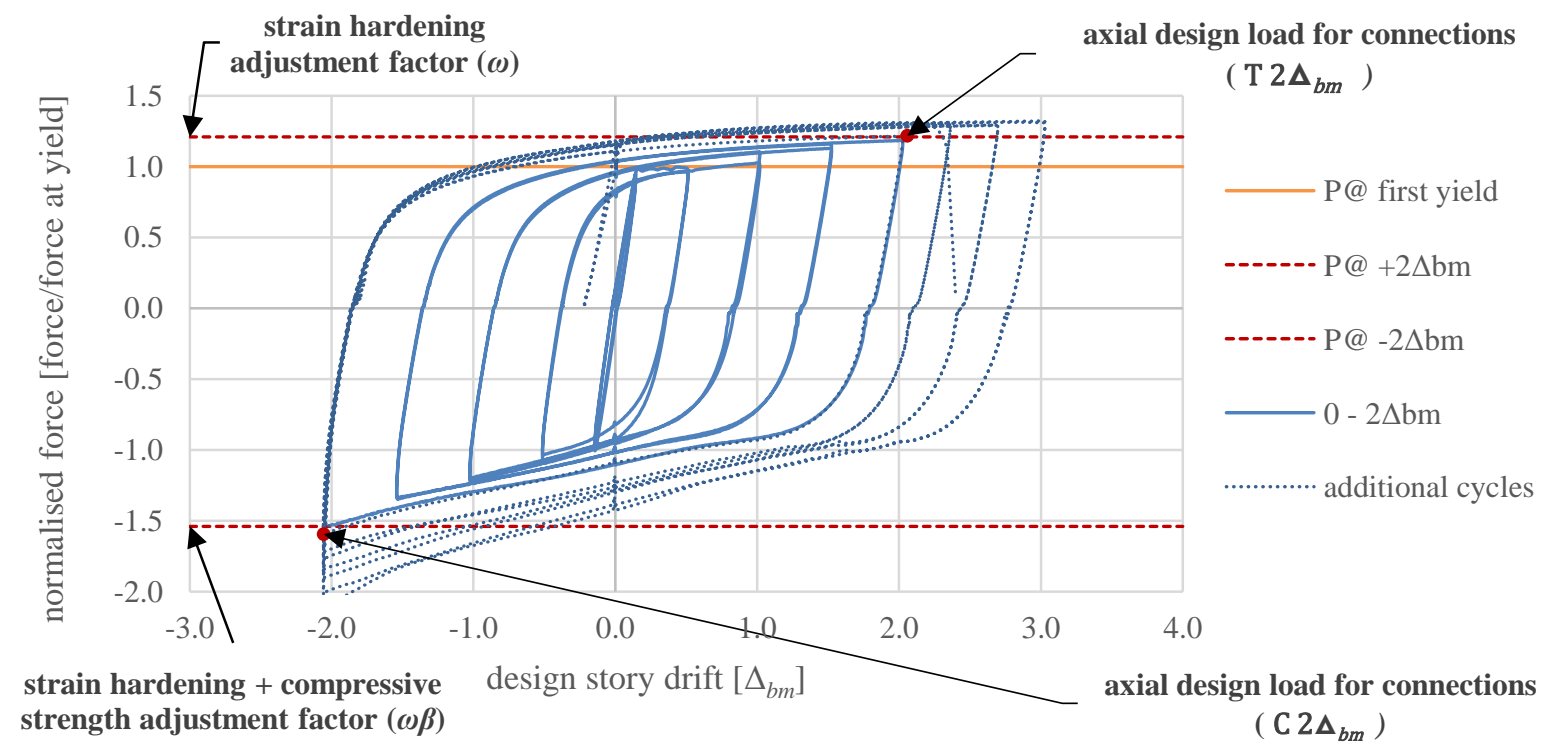

Figure 10: Example of a pre-qualification BRB test results and how over strength factors are determined. 


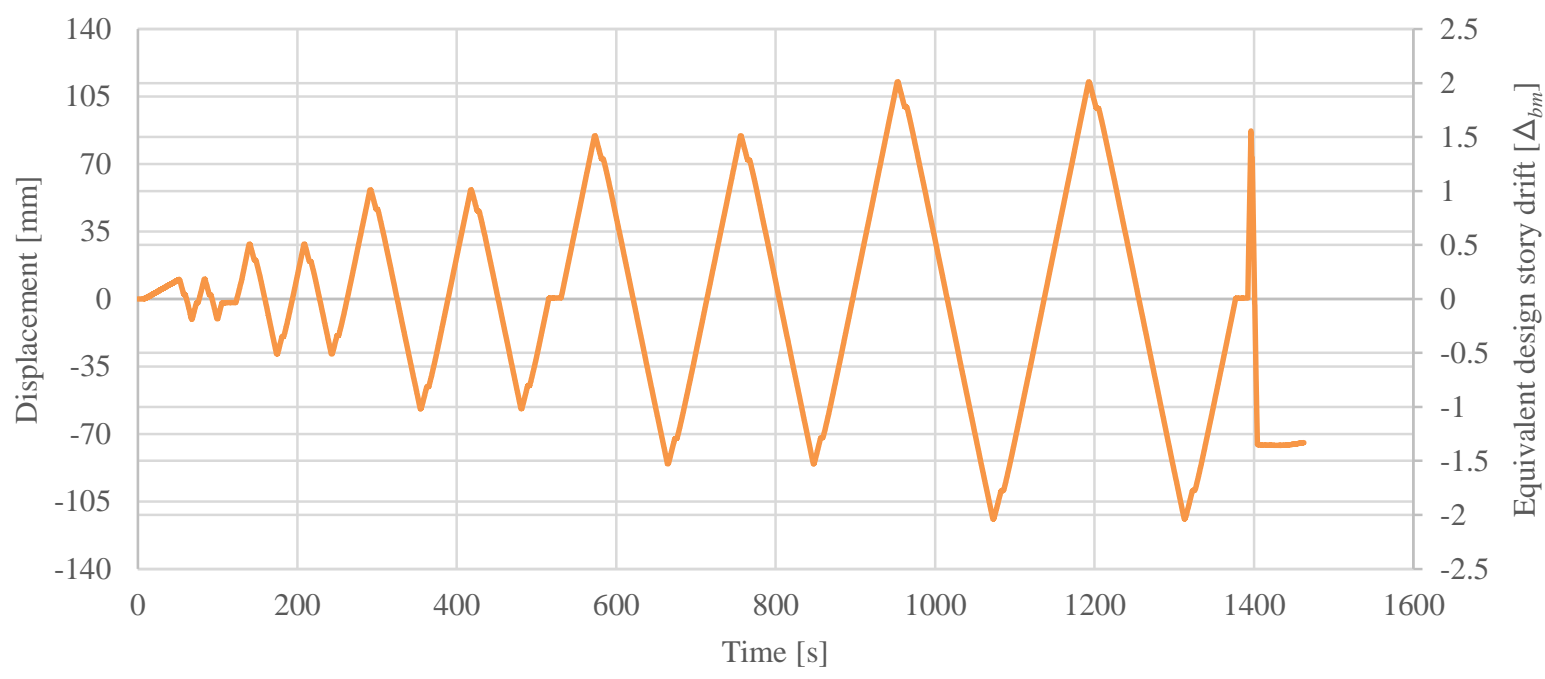

Figure 11: Loading protocol for BRB 6.5.

Equivalent design story drift $\left[\Delta_{b m}\right]$

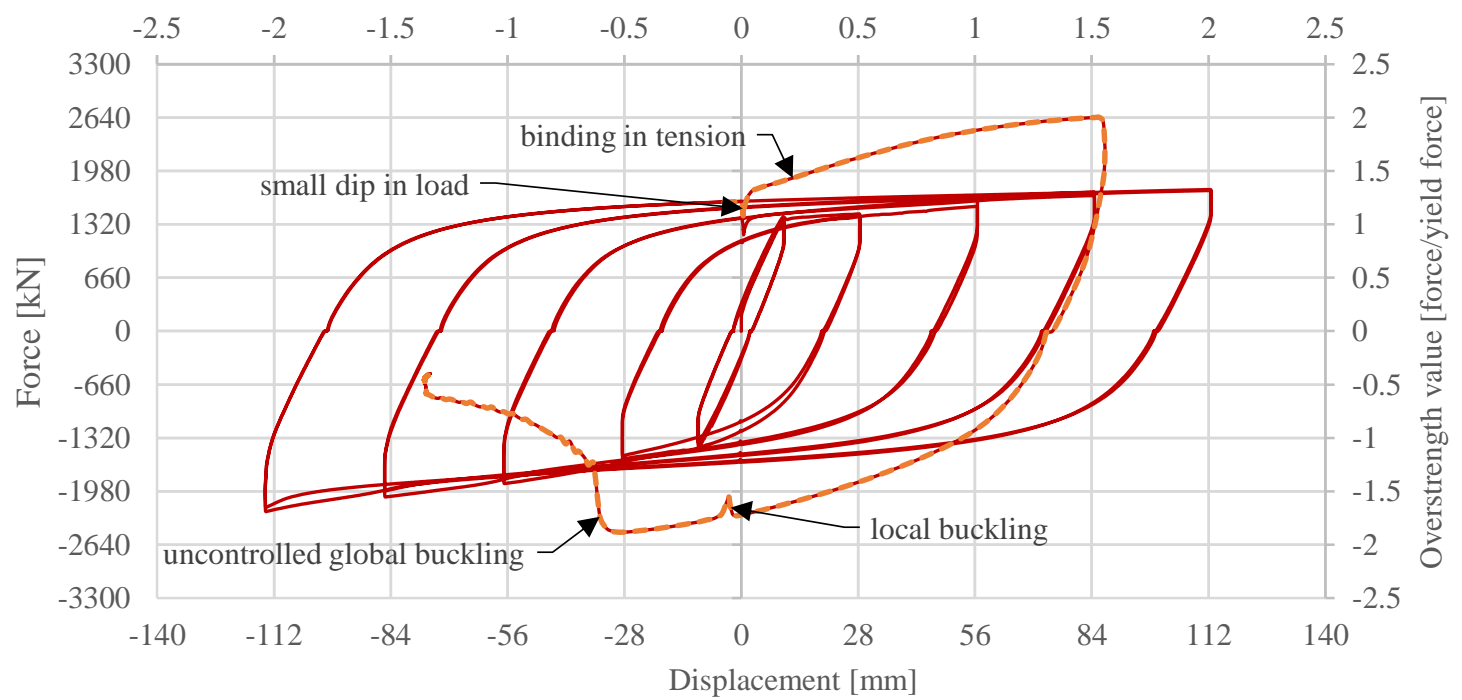

Figure 12: Experimental results for BRB 6.5.

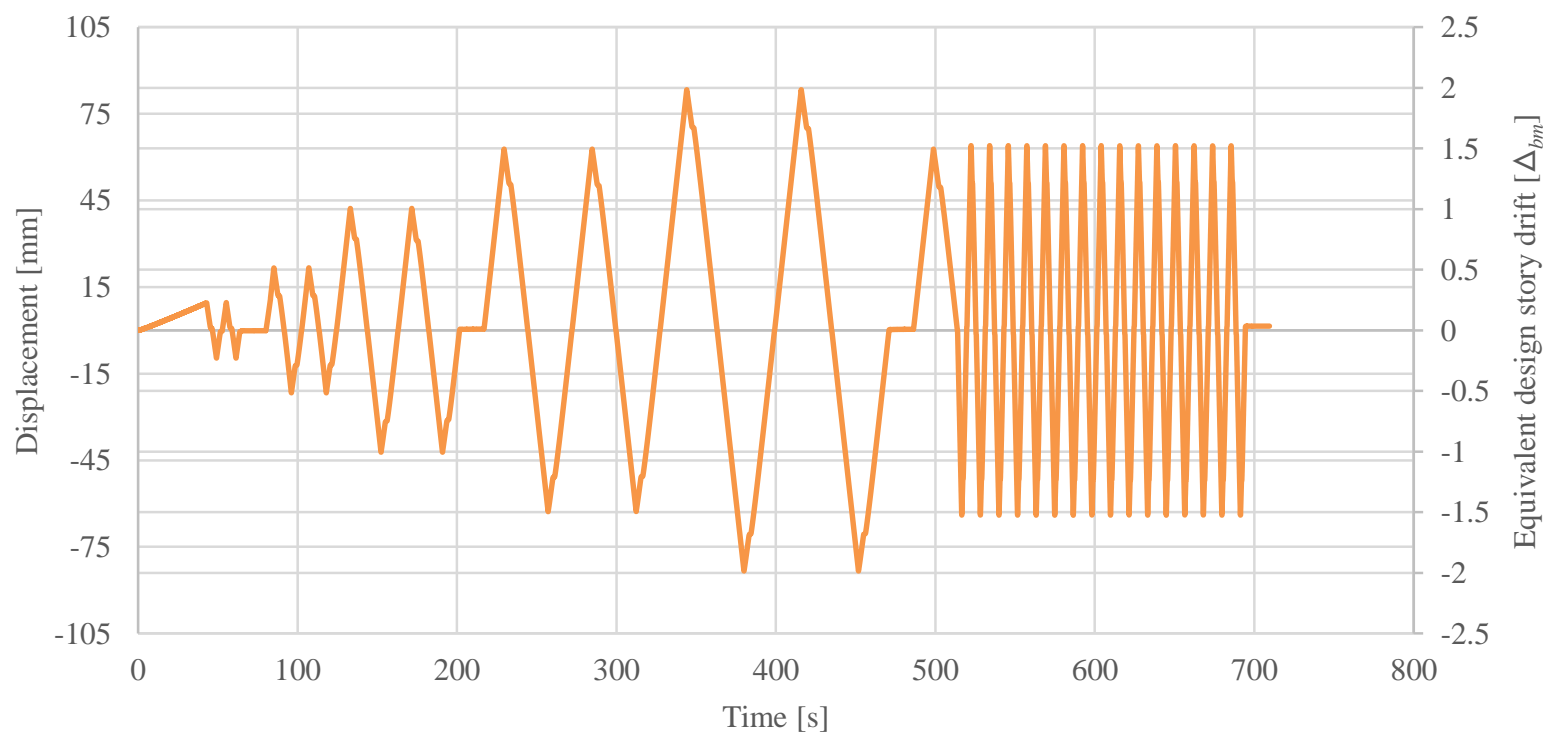

Figure 13: Loading protocol for BRB 6.5a. 


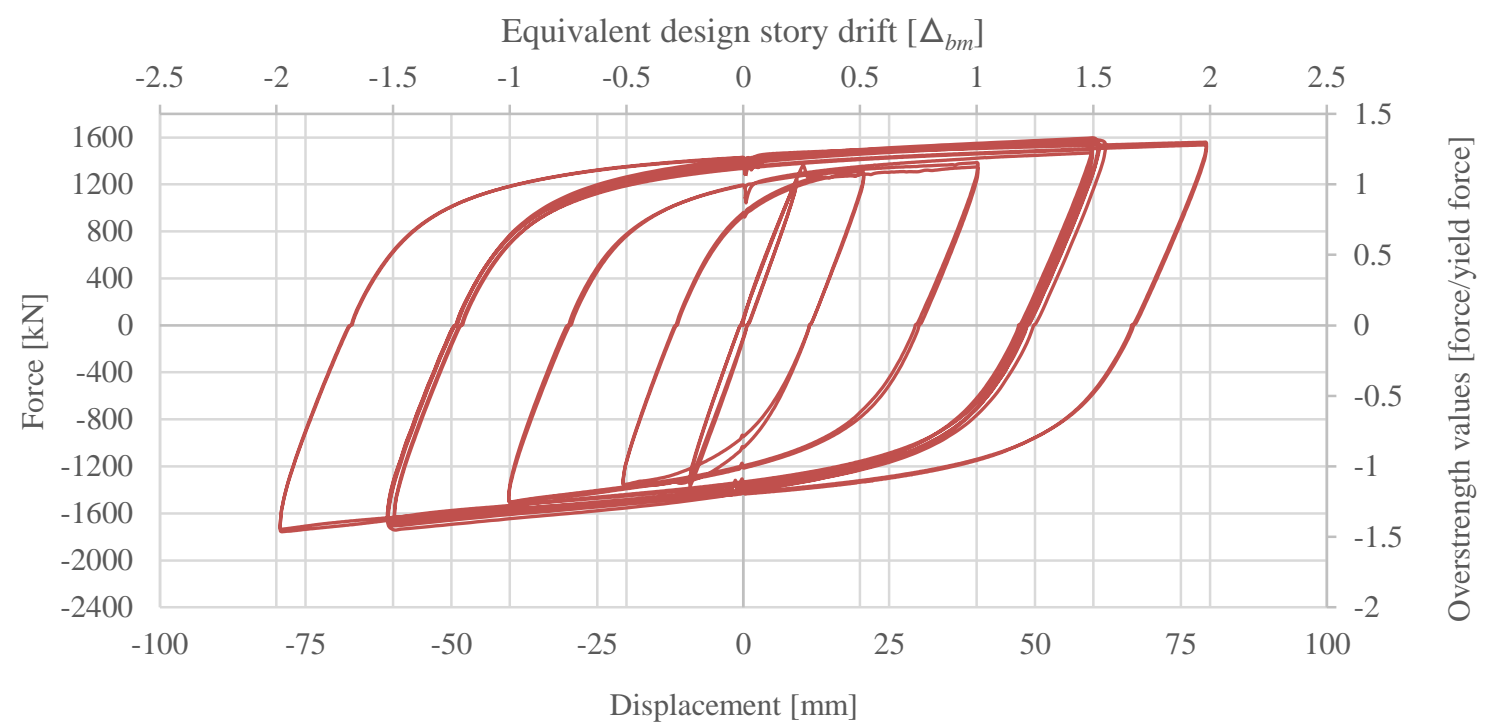

Figure 14: Experimental results for BRB 6.5a.

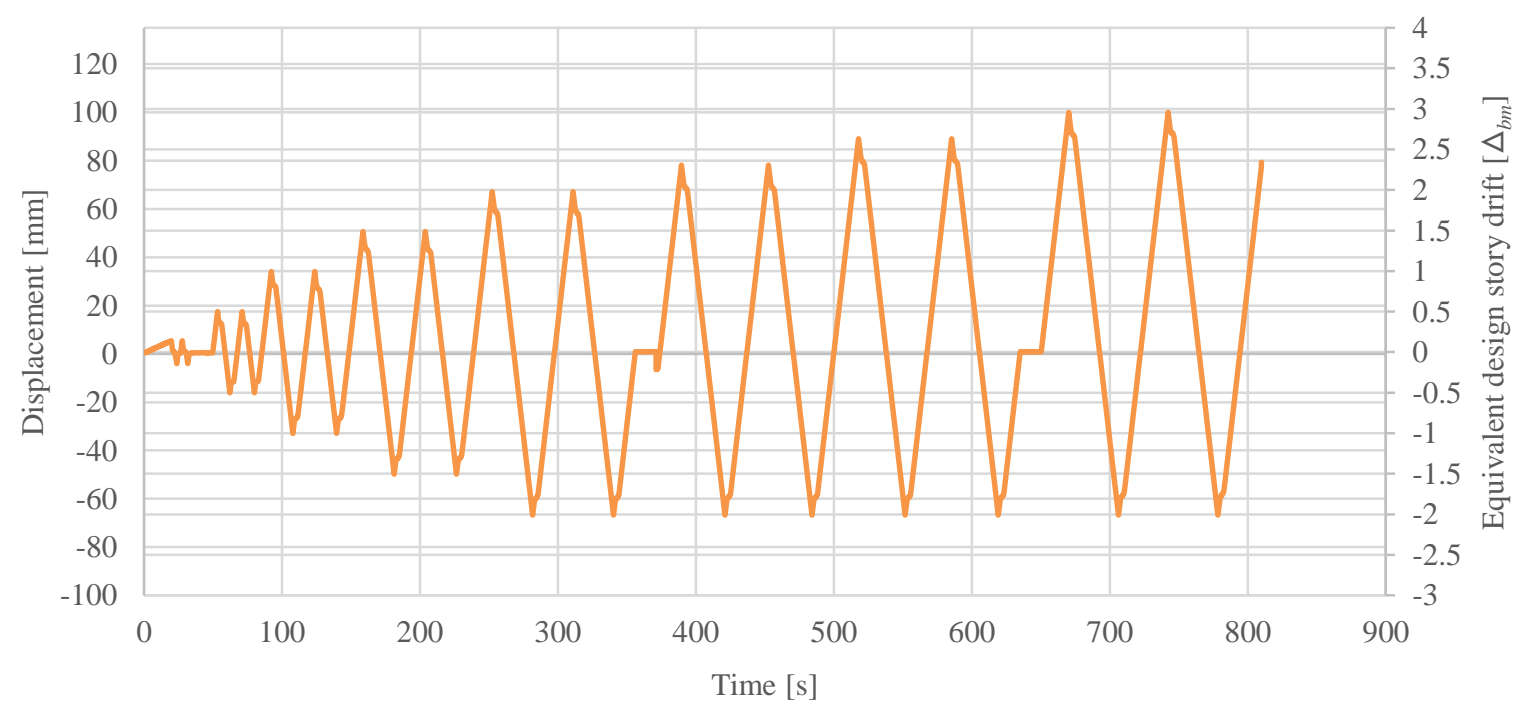

Figure 15: Loading protocol for BRB S3.

Equivalent design story drift $\left[\Delta_{b m}\right]$

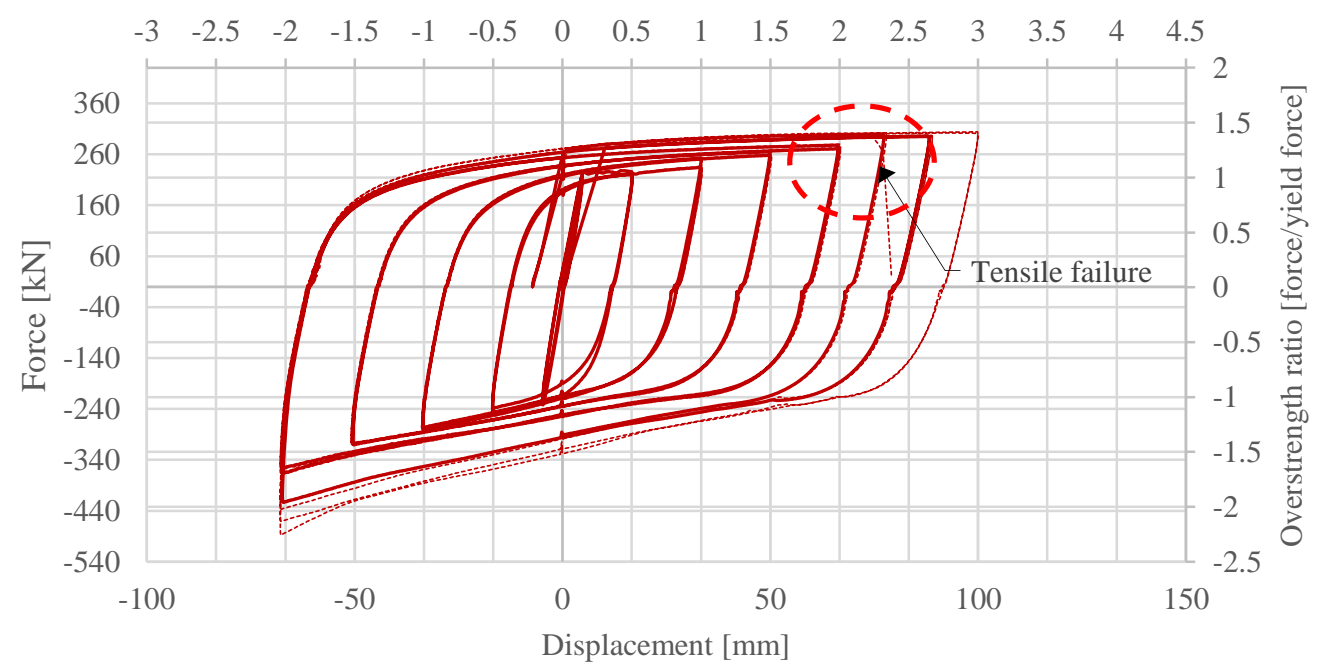

additional loading

ANSI-341 loading protocol

Figure 16: Experimental results for BRB S3. 


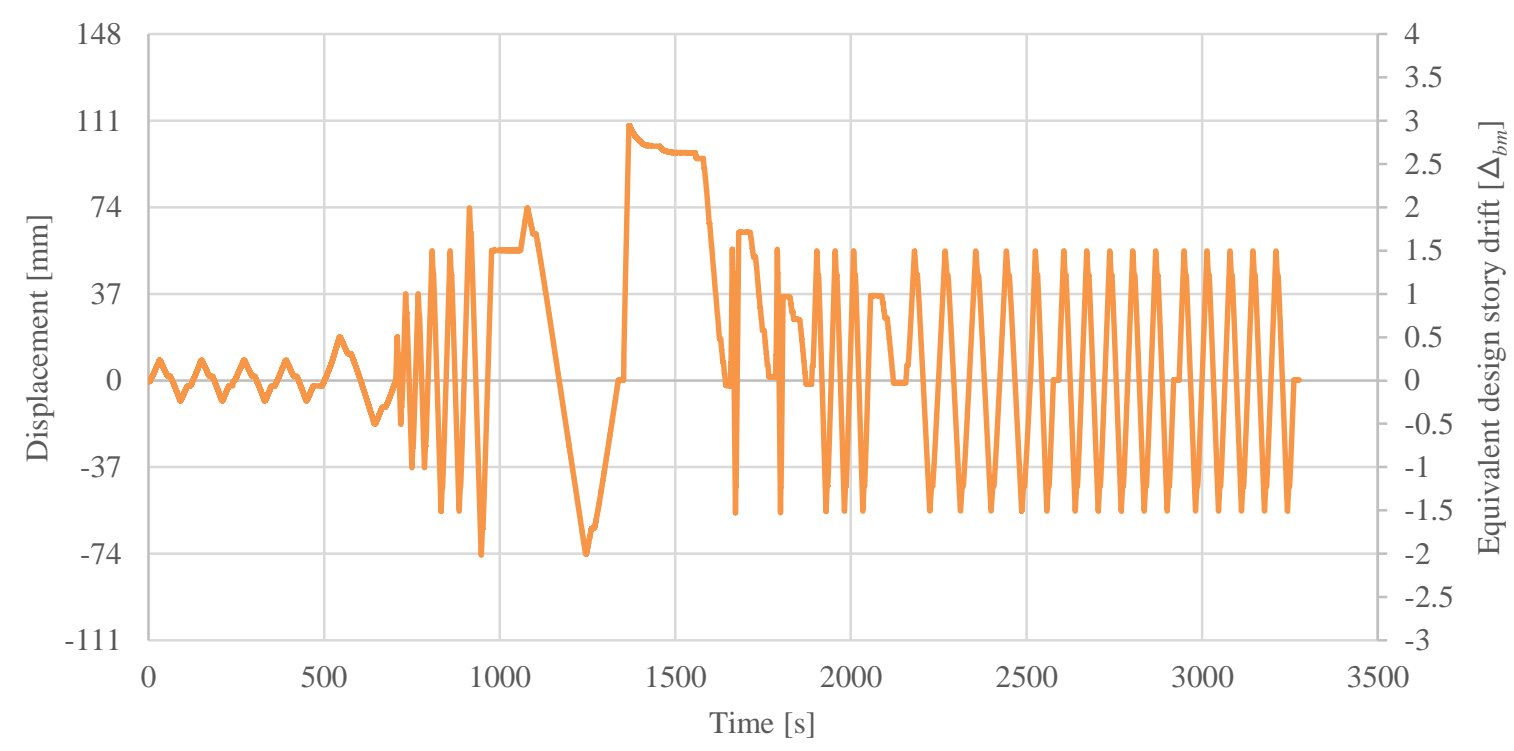

Figure 17: Loading protocol for BRB C6.

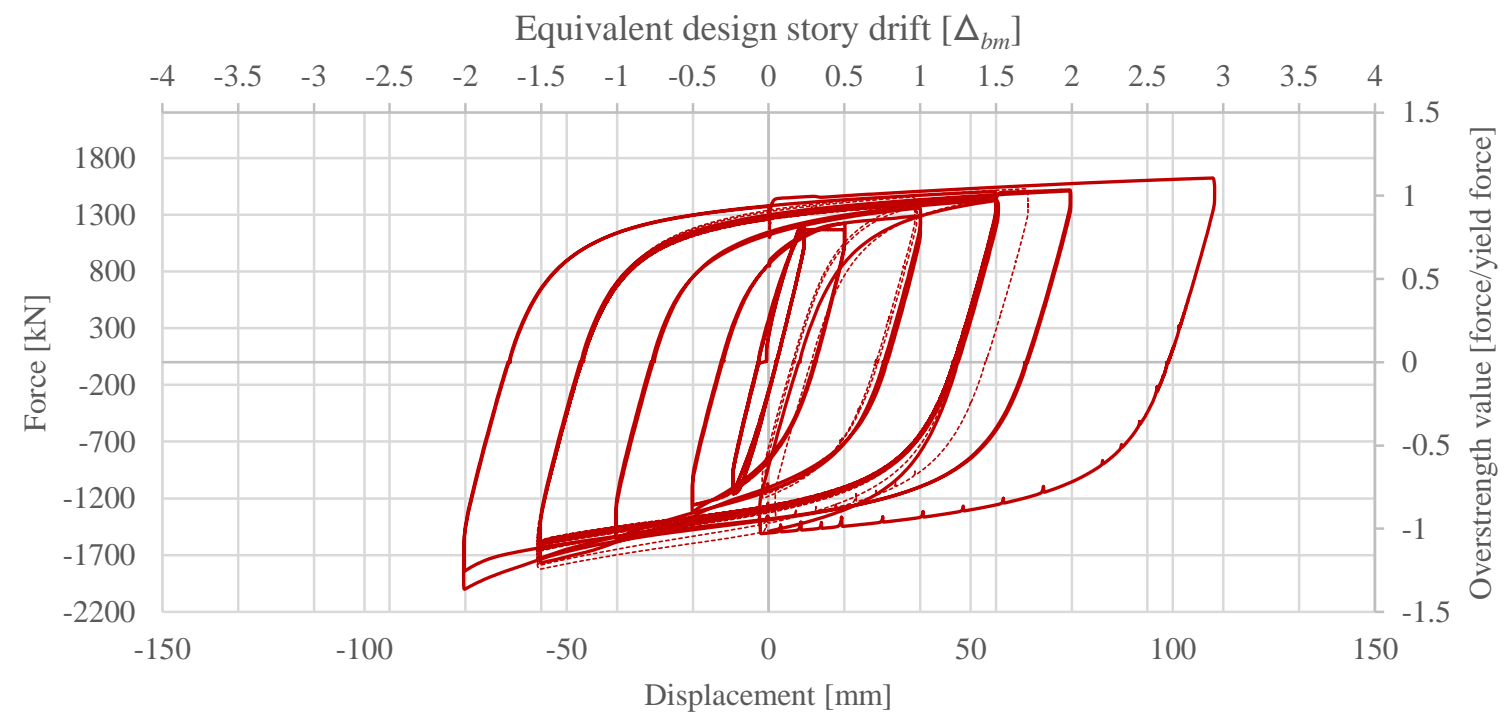

ANSI-341 loading protocol -.----o----.- additional loading

Figure 18: Experimental results for BRB C6.

\section{POST TEST OBSERVATIONS}

Following testing of the three brace types presented in Table 1, it is of interest to compare results and observations between the different specimens.

\section{BRB 6.5}

This brace displayed stable hysteretic behaviour up to $\pm 2 \Delta_{b m}$. This corresponds to an average strain of $2.3 \%$. The specimen required additional cycles to achieve the cumulative strain requirement of 200 times yield displacement. However, upon the first additional cycle it failed.

The failure mode was consistent with global buckling. Initially, the core seems to catch or bind inside the restrainer causing a spike in the load response during the tension cycle. Upon load reversal, this occurs again in compression, quickly followed by a degradation in stiffness associated with buckling collapse. Figure 19 shows the brace after testing with the global buckling shape clearly visible.

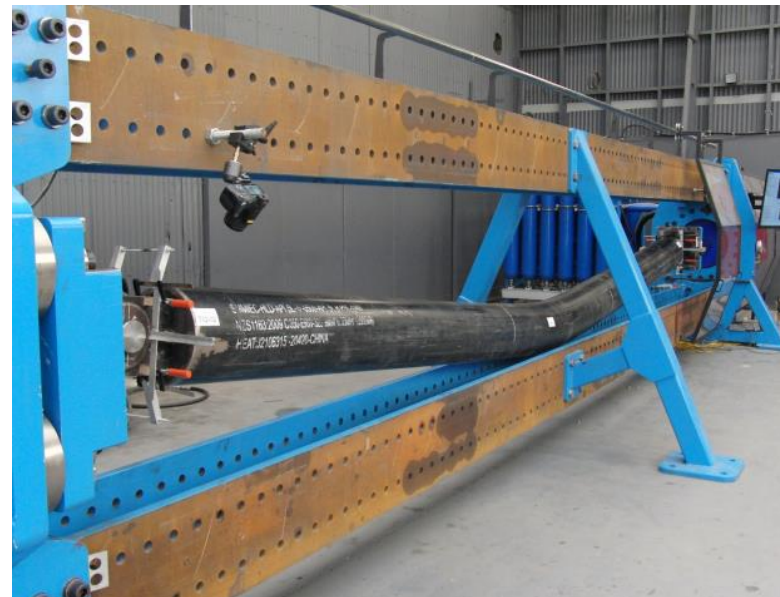

Figure 19: Global buckling in pre-qualification testing of BRB 6.5. 
The hysteresis in Figure 12 supports this behaviour by a sharp reduction in stiffness just prior to the peak load in the final compression cycle. Deconstruction of the specimen took place after testing. By cutting into the restrainer, it was revealed the core of the BRB was not running through the direct center of the restrainer. Figure 20 and Figure 21 shows the location of the core relative to the restrainer.

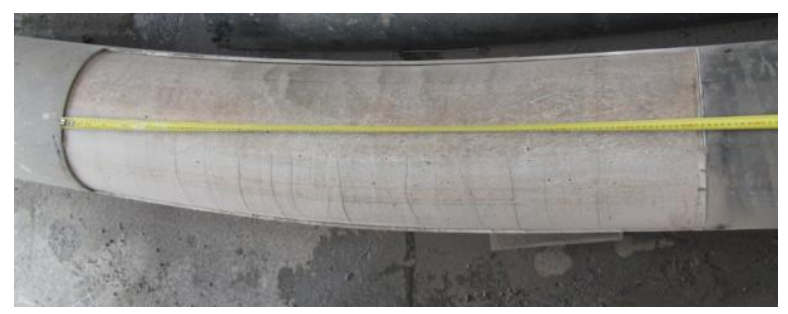

Figure 20: Post-test examination of BRB 6.5 showing cracking underneath steel casing.

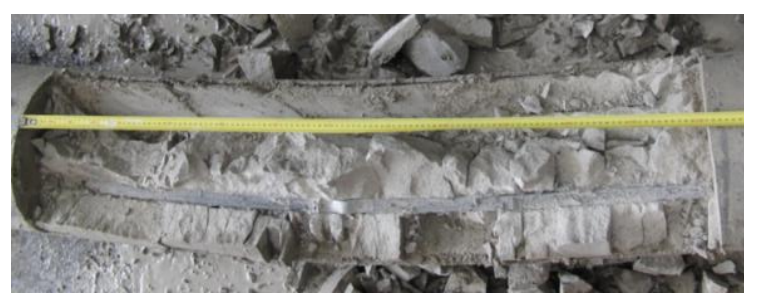

Figure 21: Post-test examination of BRB 6.5 showing location of BRB core inside restrainer.

One theory for this occurring is that during construction the brace was orientated horizontally, allowing gravity to act along the length of the steel tendon. This caused the core to sag while the grout cured inside the restrainer. It is also possible that injection of the viscous grout further pushed the core to one side. This failed test lead to a review of the construction process and improvements to ensure the core remains in the center of the BRB during fabrication of each BRB. With these steps added to the fabrication process, the same design, intended for a different frame was tested at a later date. Results showed this brace to perform well and able to meet all the acceptance criteria set out in ANSI/AISC 341-16. Full cycles to a displacement corresponding to $2 \Delta_{b m}$ were achieved. This corresponds to an average strain (displacement/core yield length) of $1.7 \%$.

\section{BRB S3}

This brace achieved the full loading protocol set out in ANSI/AISC 341-16 without core rupture or any signs of progressive reduction in load carrying capacity. When subjected to further loading cycles, BRB S3 continued to respond with increased peak load for each increased displacement target until failure occurred. Displacement amplitudes corresponding to $3 \Delta_{b m}$ in tension and $2 \Delta_{b m}$ drift in compression were achieved. This corresponds to an average strain of $3.7 \%$. Upon initiation to a target displacement corresponding to 3.5 times the design story drift in tension, core rupture was observed. This is confirmed by a sudden loss of load carrying capacity and an audible noise typical to that of a steel tensile failure. This is in agreement with hysteresis plot in Figure 16. Deconstruction of this specimen did not take place after testing.

\section{BRB C6}

Lastly investigated is BRB C6. This brace also achieved the full loading protocol as set out in ANSI/AISC 341-16 without core rupture or any signs of progressive reduction in load carrying capacity. Cycles reaching a displacement corresponding to $3 \Delta_{b m}$ in tension and $2 \Delta_{b m}$ in compression were achieved. This corresponds to an average strain of $2.6 \%$. This specimen was also subjected to additional cycles at $1.5 \Delta_{b m}$ resulting in cumulative inelastic axial ductility of 650 times displacement at yield strain without failure.

Upon completion of testing of BRB C6, the specimen was removed from the test apparatus and deconstructed to expose the BRB core. With the steel casing removed, several cracks within the grout sleeve were discovered. Two longitudinal cracks were present, as well as a multitude of circumferential cracks as can be seen in Figure 22. Upon removal of the grout sleeve no observable defects to the core were visible.

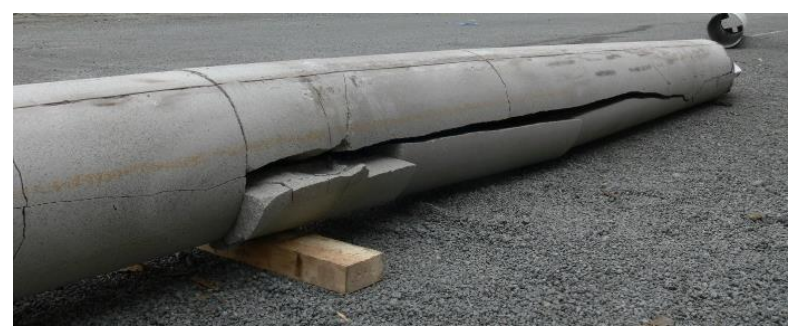

Figure 22: Specimen C6, observed cracks in grout with outer shell removed.

\section{Comparison of Overstrength Factors}

The post-yielding strength determines load adjustment factors, obtained from pre-qualification testing (as per Figure 10). In tension, the design load for connecting elements is the product of the core area, yield strength and is multiplied by a strain hardening adjustment factor. In compression, a strain hardening and a compressive adjustment factor are multiplied by the core area and yield strength. Upon comparing the hysteresis plots between each brace, in was found that BRB S3, BRB C6 had a higher compression adjustment factor $(\beta)$ compared to $\mathrm{BRB}$ 6.5. According to ANSI/AISC 341-16, this is acceptable if the ratio between tension and compression cycles does not exceed 1.3 .

\section{Debonding Material}

A key feature to each BRB is the debonding material between the core and the concrete restrainer. The debonding material is a thin film-like material that provides a low friction sliding interface between the core and the restrainer. This interface minimizes axial load transfer between the core and the restrainer. The thickness can vary depending on the debonding material used, but is generally $2.0 \mathrm{~mm}$ and runs the entire length of the restrainer. During compression cycles, significant normal forces develop at this interface allowing the core to deflect in either direction. The actual behaviour of the debonding material deforming inside the restrainer is very complex. The material heats up due to the plasticity of the steel core. This combined with the bending and stretching behaviour of the core, forces the material in and out of the space between the restrainer and the core in a fluid-like way. Physical observations of the material extruding out of the ends of the restrainer support this. Specific properties of the debonding material in commercial $\mathrm{BRBs}$ are generally confidential. However, information from the supplier of the braces detailed in this paper suggest it is a type of butyl rubber. Figure 23 shows how the debonding material has extruded from the end of the restrainer. 


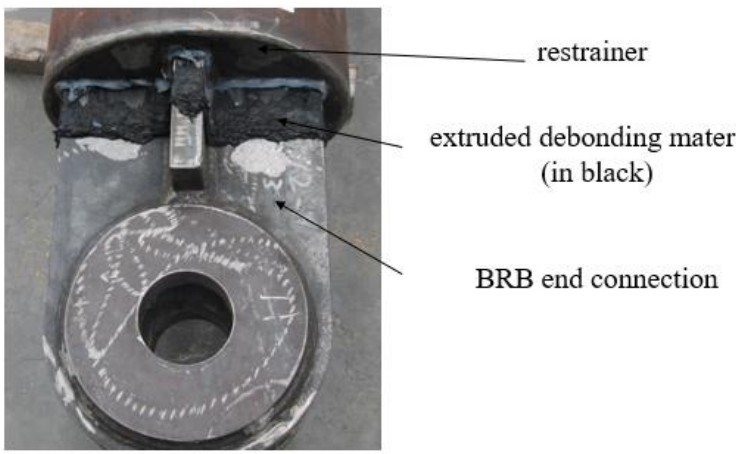

Figure 23: Post-test observation of debonding material.

\section{ROLE OF FINITE-ELEMENT-MODELLING}

BRBs became popular in New Zealand following the 2010/2011 Christchurch earthquakes. Testing in New Zealand has been generally limited to uniaxial loading. Brace prequalification testing should show a BRB design and manufacturing are adequate but does not demonstrate full frame stability and cannot accurately predict when and where plastic hinges occur as a result of realistic earthquake loading such as bi-directional displacements, rotations and interaction of the BRB with frame elements. This is motivation for subassemblage testing and/or stability modelling of each bespoke design in addition to pre-qualification testing.

High fidelity modelling paired with pre-qualification testing is an approved alternative to meeting the requirements of ANSI/AISC 341-16. This can help engineers verify designs including retro-fit projects, irregular structural systems or large BRB designs. Current computer technology and non-linear FEA tools enable all the significant mechanisms in deforming BRBs to be captured. This type of modelling enables detailed analysis of stress-strain behaviour, along with global loaddisplacement history predictions.

As BRBs are a proprietary component, structural engineers treat the inner working of a BRB as a 'black box' with the end conditions often treated as ideal axial load connections. A key challenge with experimental testing is trying to observe the deforming behaviour of the core as it interacts with the restrainer, and measuring the additional rotational and flexure demands this creates. Results should then influence the type of connection detailing specified. The current tools available for connection design, namely guidance from international groups, information from suppliers and recorded test data, either do not agree, are vague, limited or not applicable to NZ application. High fidelity modelling is an attractive option to study the stress-strain behaviour of BRBs to ensure safe BRB and connection design. An example of high fidelity modelling is shown in Figure 24. Details of the modelling approach are described elsewhere [20].

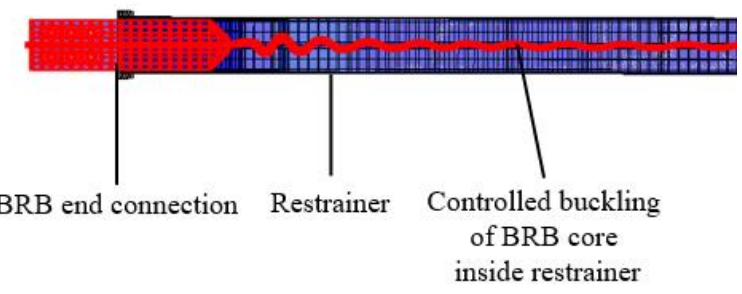

Figure 24: Realistic simulation of BRB S3, (deformation scaled to show higher mode buckling).

Such modelling can supplement pre-qualification BRB test data. Any BRB variant can be modelled, requiring only adjustments for material and geometric data.

To demonstrate the capability of such modelling. Figure 25 shows the numerical and experimental results for each BRB type. Each BRB varies in size, connection type, capacity and design load and close replication is observed to occur in all three BRB types.

As previously stated, one observation between the BRBs test results presented in this paper was that the compressive overstrength ratios were more prominent in BRB S3 and BRB C6 compared to specimen BRB 6.5a. FEA modelling results were used to investigate this, and it was found that the relative lengths of the polystyrene spacer is the main contributing factor to the differences in compressive overstrength ratios. The length of the spacer used in BRB S3 and BRB C6 was 50\% less than that used in BRB 6.5a. A shorter spacer means more axial load is transmitted to the restrainer as the transition zone of the BRB core pushes up into the void. This action occurs at each end, so as compressive displacements increase, the restrainer begins to contribute to a higher compressive load. This idea is supported by the FEA models that show the stress concentration in this zone when the spacer pushes against the restrainer. Figure 26 shows the stress concentration is higher for both BRB $\mathrm{S} 3$ and BRB C6 when displacements $=-2.0 \Delta b m$, compared to BRB 6.5a, where grey and red indicate the highest levels of stress and blue indicates low levels of stress.

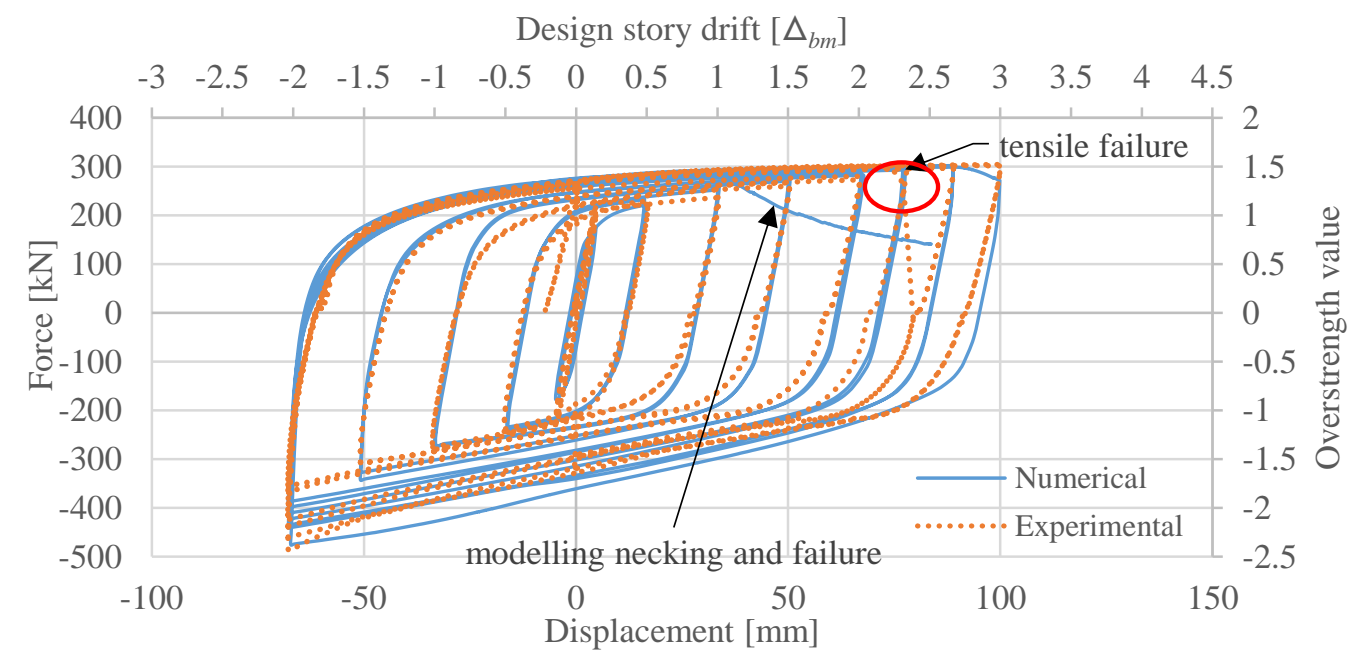

(a) BRB S3 


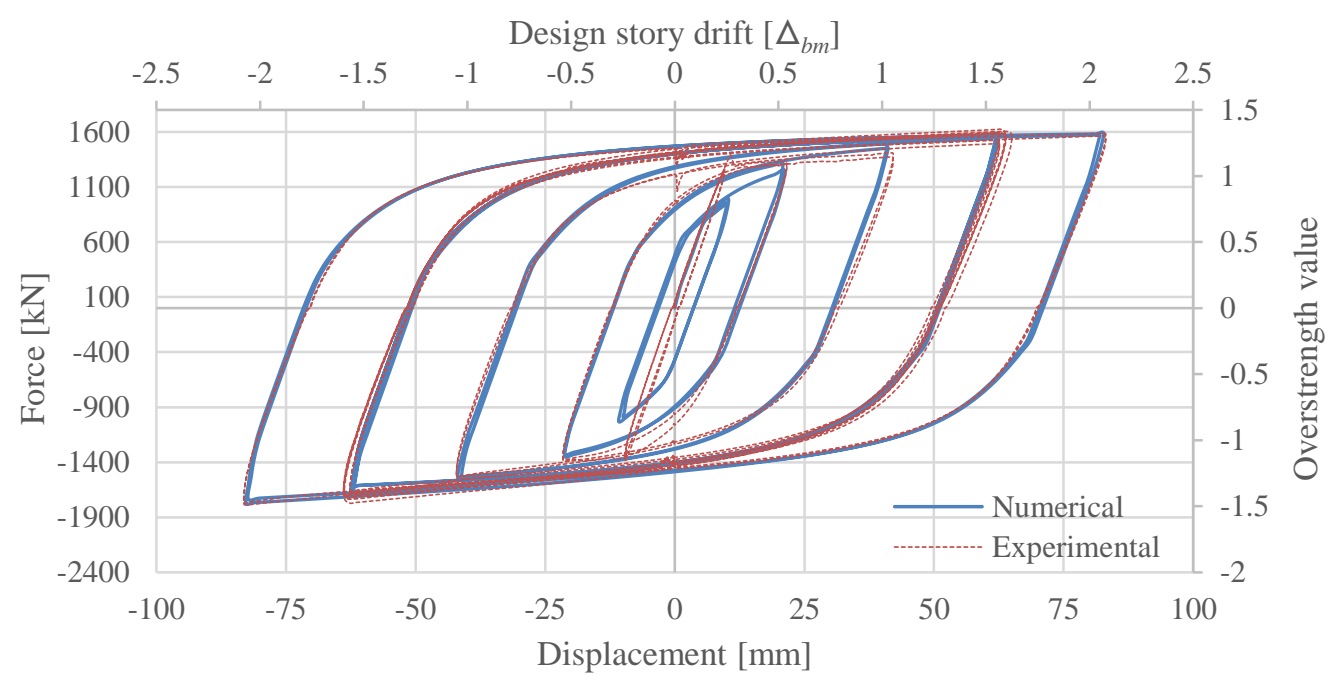

(b) BRB 6.5a

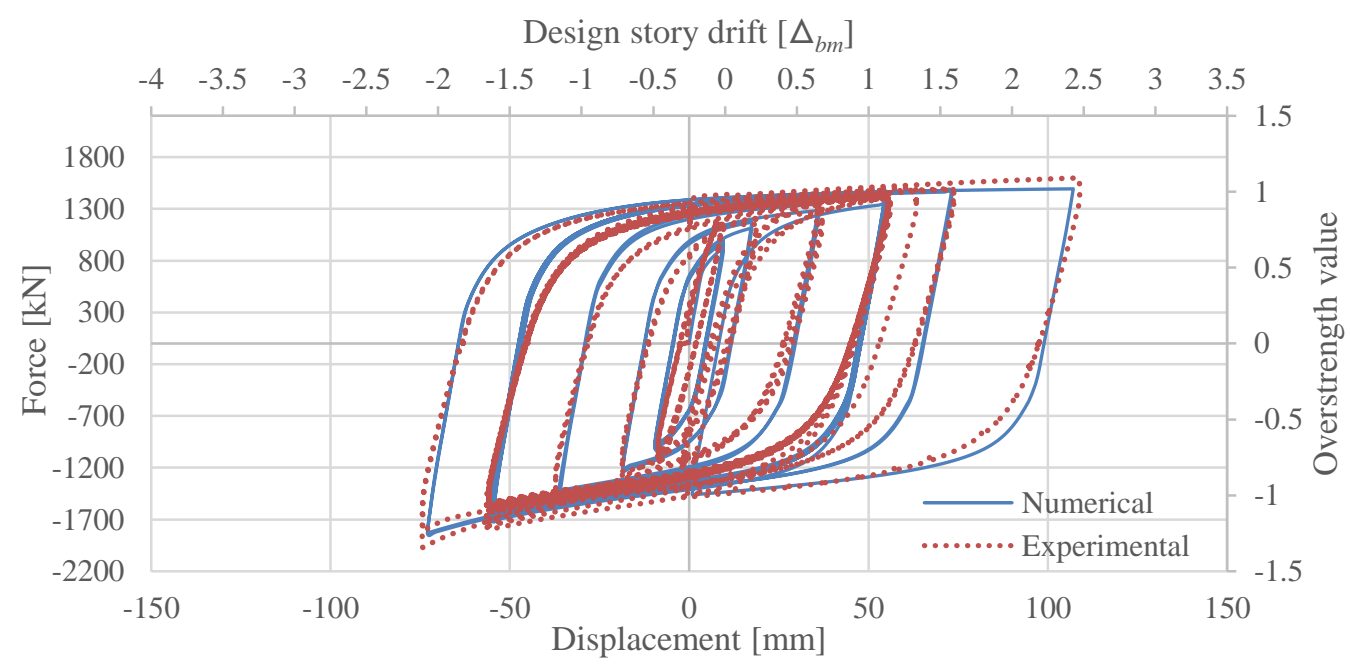

(c) BRB C6

Figure 25: Numerical and experimental hysteresis.

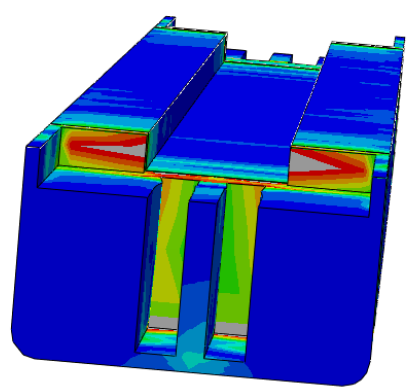

(a) BRBS3

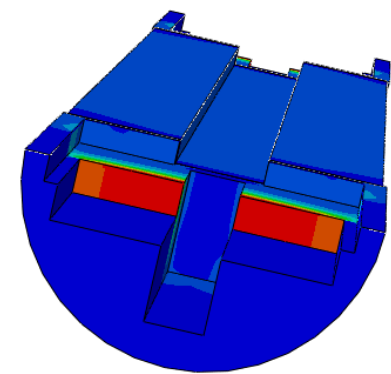

(b) $B R B C 6$

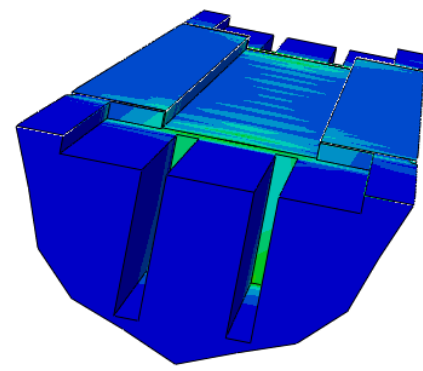

(c) BRB 6.5a

Figure 26: Von Mises stress in the concrete restrainer of each BRB (displacement $\left.=-2.0 \Delta_{b m}\right)$.

The overstrength value for each cycle is calculated by dividing the peak compression force by the peak tensile force. Close examination of the peak forces in Figure 25 show how accurate the simulations are at predicting overstrength values. Numerically, the overstrength values were found to be within $8 \%$ of the experimental results. This suggests the FEA modelling strategy can be used to predict cyclic BRB behaviour. Specifically, the overstrength ratio at $2.0 \Delta_{b m}$, which is used to generate overstrength factors for building design, are within $6 \%$ of experimental data.
These models represent a strategy [20] for modelling generic $\mathrm{BRBs}$ requiring only shop drawing and cyclic material test data. Each model captures the complex behaviour of the BRB under cyclic loading, where contact, higher mode buckling, and nonlinear material properties are present. Evidence of other BRB models built using this strategy can be found in [21]. The process of selecting the appropriate material and contact modelling parameters, the sensitivity of these parameters along with techniques used to overcome the computational demands, numerical instabilities and convergence issues related to this problem are described elsewhere [20]. 
Evidence presented suggest the overall modelling approach described here can be used to study and better understand the local and global behaviour of a BRB. By adjusting the boundary conditions, adding frame elements (which have relatively simple geometry and no contact conditions) and varying the direction of loading we can simulate full-frame or subassemblage testing.

The type of modelling presented in this paper also gives us clues about what may be occurring inside the restrainer. For example, Figure 27 presents snapshots of the strain profile at the outer edge along the length of the core for a series of cycles at peak tensile and compressive displacement loads. The graph indicates that friction mutes the compressive strains at core mid-length and that high levels of strain (up to 30\%) occur closer to the ends at high load when constrained buckling is predicted to have a strong influence on axial load distribution and peak strains. This suggests why the failure in BRB S3 may have occurred when average strain along the length was only $3.7 \%$.

In addition, the failure exhibited in BRB S3 gives one opportunity to investigate the capability of the modelling strategy to capture tensile failure as localized necking. Figure 28 shows the necking behaviour captured in the BRB S3 model as associated with Figure 16. One small measure of validation is that this adjusted material model (calibrated to the failure of
BRB S3) did not result in necking failures of other BRBs tested, corresponding with observed behaviours.

Although this demonstrates the ability to capture necking failure, this is clearly not a validated capability, as it is unknown whether the predicted location of failure in BRB S3 shown in Figure 28 is correct, and no blind predictive capability has been conducted. Proper development of a failure prediction capability would require a combination of cyclic uniaxial testing and BRB testing that somehow collects data on the spatial variation in strain along the core. This poses a significant challenge as, for example, traditional methods for fastening strain gauges are likely to not survive. Moving forward, the overall modelling approach described here shows the potential to augment pre-qualification testing to meet requirements of ANSI/AISC 341-16.

\section{CONCLUSIONS}

In New Zealand, there is currently no standard or regulation on how to incorporate BRBFs in a commercial building design. This requires building designs (with BRBFs) to take part in a peer review to gain building compliance, and requires evidence from testing to support the design. Details from the American standard (ANSI/AISC 341-16) that explain how and why this evidence is important is discussed. Specifically, ANSI/AISC 341-16 prescribes two forms of testing.

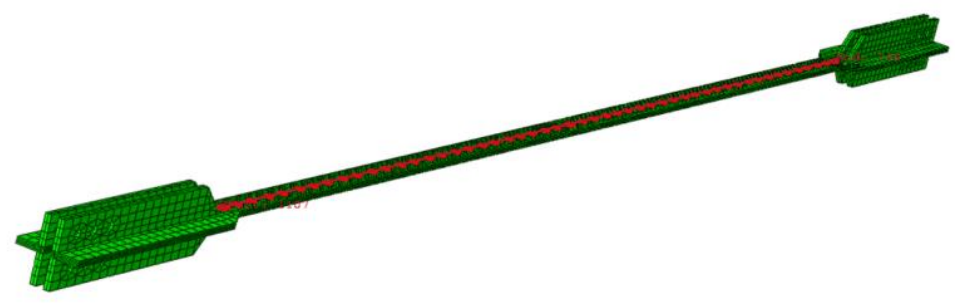

(a) Path of elements where strain was recorded

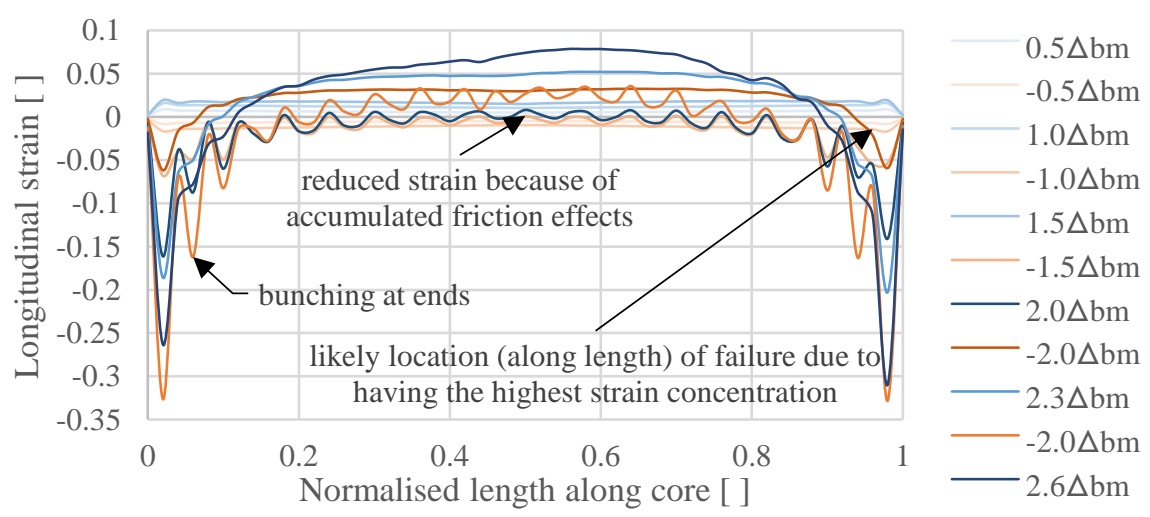

(b) Plot of strain profile

Figure 27: Strain recordings along core at first cycle of each target displacement (BRB S3).

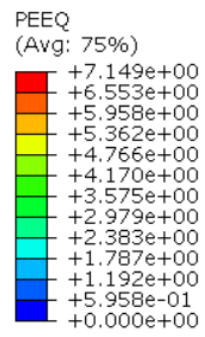

Location of failure caused by necking

Figure 28: Location of failure caused by necking in model of BRB S3. 
1) Brace pre-qualification testing is conducted to ensure each BRB specimen is suitably designed and manufactured to meet a buildings; earthquake design limits. This type of testing isolates the performance of a BRB by applying a cyclic uniaxial loading protocol.

2) Sub-assemblage testing investigates the performance of the entire BRB frame system, specifically to ensure stability is maintained during design level earthquake events.

However, as ANSI/AISC 341-16 is not a governing document in New Zealand, the recommendations are often made to fit within the constraints of a building project. By way of example, this paper presents the experimental test process and results for three different BRBs. The first BRB 6.5 test showed pre-mature failure. Post-test observations confirmed manufacturing errors led to global buckling failure and highlights the need for strict quality control during fabrication. Each remaining BRB specimen achieved the full loading protocol (equivalent displacement up to two times the design story drift) without core rupture or any signs of progressive reduction in load carrying capacity, thereby meeting all the acceptance criteria stipulated in ANSI/AISC 341-16 Section K. This information is valuable since test data for BRBs relevant to the New Zealand construction environment are not commonly available, with many experiments in New Zealand restricted to relatively small braces.

The capability of high fidelity modelling which captures the complex behaviour under cyclic loading of contact conditions, higher mode buckling, and material non-linearity is also discussed. Close replication of FEA simulations to physical test data was observed to occur in all three BRB types. The simulated results predicted the overstrength ratio for all cycles within $8 \%$, and with predictions at $2.0 \Delta_{b m}$ being within $6 \%$ for all three BRB designs [20]. This form of modelling can be used to supplemental the physical testing required to gain building compliance and to study the stress-strain behaviour of the BRB, in particular the deformation of the BRB core inside the restrainer.

\section{REFERENCES}

1 Kimura KY and Takeda T (1976). "Tests on braces encased by mortar in-filled steel tubes". Summaries of Technical Papers of Annual Meeting, Architectural Institute of Japan, pp. 1041-1042. (in Japanese)

2 Rabbat BG and Russell HG (1985). "Friction coefficient of steel on concrete or grout". Journal of Structural Engineering, 111(3): 505-515.

https://doi.org/10.1061/(ASCE)07339445(1985)111:3(505)

3 Watanabe A, Hitomi Y, Yaeki E, Wada A and Fujimoto M (1973). "Experimental study of elasto-plastic properties of precast concrete wall panels with built-in insulating braces". Summaries of Technical Papers of Annual Meetingy, Architectural Institute of Japan, pp. 1041-1044. (in Japanese)

4 Bruneau M, Uang C-M and Sabelli R (2011). "Ductile design of buckling-restrained braced frames" pp. 651-687 in Ductile Design of Steel Structures, 2nd Edition, McGraw Hill, New York.

5 AISC (2016). "Seismic Provisions for Structural Steel Buildings - ANSI/AISC". American Institute of Steel Construction (AISC).

6 CEN (2010). "European Committee for Standardization (CEN) EN15129:2010 Anti-Seismic Devices".
7 ECCS (1986). "Seismic Design, Recommended Testing Procedure for Assessing the Behaviour of Structural Steel Elements under Cyclic Loads". European Convention for Constructional Steelwork - Technical Committee 1, Vol. Structual saftey and loadings - Technical working Group 1.

8 Black C, Makris N and Aiken I (2002). "Component Testing, Stability Analysis and Characterization of Buckling-Restrained Unbonded Braces". Pacific Earthquake Engineering Research Center.

9 Dunai L, Kaltenbach L, Kallo M, Kachichian M and Halasz A (2011). "Type Testing of Buckling-Restrained Braces According to EN 15129". Budapest University of Technology and Economics.

10 Wijanto S and Clifton GC (2014). "Experimental testing and design of BRB with bolted and pinned connections". Bulletin of the New Zealand Society for Earthquake Engineering, 47(4): 264-274.

https://doi.org/10.5459/bnzsee.47.4.264-274

11 Hikino T (2016). "Experimental study on remained fatigue of used BRB (Tohoku Earthquake)". Summaries of Technical Papers of Annual Meeting, Architectural Institute of Japan. (in Japanese)

12 Tsai C-Y, Lin K-C, Tsai KC, Chen L-W and Wu A-C (2018). "Seismic performance analysis of BRBs and gussets in a full-scale 2-story BRB-RCF specimen". Earthquake Engineering and Structural Dynamics, 47(12): 2366-2389. https://doi.org/10.1002/eqe.3073

13 Khoo H-H, Tsai K-C, Tsai C-Y and Wang K-J (2016). "Bidirectional substructure pseudo-dynamic tests and analysis of a full-scale two-story buckling-restrained braced frame". Earthquake Engineering and Structural Dynamics, 45(7): 1085-1107. https://doi.org/10.1002/eqe.2696

14 Tsai K-C, Wu A-C, Wei C-Y, Lin P-C, Chuang M-C and Yu Y-J (2014). "Welded end-slot connection and debonding layers for buckling-restrained braces". Earthquake Engineering and Structural Dynamics, 43: 785-1807. https://doi.org/10.1002/eqe.2423

15 Fahnestock LA, Ricles JM and Sause R (2007). "Experimental evaluation of a large-scale bucklingrestrained braced frame". Journal of Structural Engineering, 133(9): 1205-1214. https://doi.org/10.1061/(ASCE)07339445(2007)133:9(1205)

16 Uriz P and Mahin S (2008). "Toward Earthquake-Resistant Design of Concentrically Braced Steel-Frame Structures". Pacific Earthquake Engineering Research Center.

17 Tremblay R, Bolduc P, Nevilee R and DeVall R (2006). "Seismic testing and performance of buckling-restrained bracing systems". Canadian Journal of Civil Engineering, 33(2): 183-198. https://doi.org/10.1139/105-103

18 Takeuchi $\mathrm{T}$ and Wada A (2017). "Buckling-Restrained Braces and Applications". The Japan Society of Seismic Isolation, Japan.

19 NCREE (2014). "User Guide for BOD: BucklingRestrained Brace and Connection Design Procedure". National Center for Research on Earthquake Engineering (NCREE), National Taiwan University, Taiwan.

20 Court-Patience D and Garnich M (2021). "FEA strategy for the realistic simulation of buckling-restrained braces". Journal of Structural Engineering. https://doi.org/10.1061/(ASCE)ST.1943-17 541X.0003033

21 Court-Patience D (2021). "A Numerical Study of Structural Performance of Buckling-Restrained Braces and End Connections". PhD Dissertation, Department of Mechanical Engineering, University of Canterbury, Christchurch, New Zealand. 